\title{
Bone morphogenetic protein-2 induced posterolateral lumbar fusion in a mouse model
}

\section{Honfang Chen}

Shanghai Jiao Tong University School of Medicine

\section{Mingke Guo}

NCO School of Army Medical University

\section{Lin Du}

Shanghai Jiao Tong University School of Medicine

\section{Chen Han}

Shanghai Jiao Tong University School of Medicine

Haijun Tian ( $\square$ haijuntianmd@gmail.com )

\section{Changqing Zhao}

Shanghai Ninth People's Hospital,Shanghai Jiao Tong University School of Medicine https://orcid.org/0000-0003-1090-7776

\section{Elsa J Brochmann}

VA Greater Los Angeles Healthcare System

\section{Samuel S Murray}

VA Greater Los Angeles Healthcare System

\section{Michael D Daubs}

University of Nevada School of Medicine

Jeffrey C Wang

University of Southern California

\section{Research article}

Keywords: lumbar fusion model, mouse, Orthopedics

Posted Date: July 22nd, 2020

DOl: https://doi.org/10.21203/rs.3.rs-46978/v1

License: (c) (1) This work is licensed under a Creative Commons Attribution 4.0 International License. Read Full License 


\section{Abstract}

Objective: The goal of this study was to establish a rhBMP-2 induced posterolateral intertransverse lumbar fusion model in the mouse to serve as an acceptable substitute for models using larger animals, such as the rat, by defining the pertinent anatomy and establishing an effective dosing regimen in the mouse.

Methods: Forty 10-week-old male Institute of Cancer Research (ICR) mice were randomized into five groups and were subjected to posterolateral lumbar fusion surgery at the L5-L6 level and received the following assigned treatment regimens: Group A: carrier collagen sponge only; Group B: $0.05 \mu \mathrm{g}$ rhBMP-2 on a collagen sponge; Group C: $0.15 \mu \mathrm{g}$ rhBMP-2 on a collagen sponge; Group D: $0.5 \mu \mathrm{g}$ rhBMP-2 on a collagen sponge; and Group E: $1.5 \mu \mathrm{g}$ rhBMP-2 on a collagen sponge. The study was ended at eight weeks post-operation and standard $\mathrm{x}$-ray and micro-computed tomography imaging were performed in addition to histological examination of dissected spine specimens.

Results: BMP-2 induced new bone at all dose levels in a dose-related manner. The mice implanted with rhBMP-2 at the dose of $0.5 \mu \mathrm{g}$ demonstrated more bone formation and a higher fusion rate compared to mice receiving lower doses. More abundant bone formation could be induced by higher dose regimen, which could also result in poorer bone quality.

Conclusions: Posterolateral lumbar fusion at the L5-L6 level can be successfully accomplished in mice. The dose of $0.5 \mu \mathrm{g}$ of rhBMP-2 delivered on a collagen sponge appears to be the optimal regimen to produce the most satisfactory fusion results.

\section{Introduction}

Since its approval by the United States Food and Drug Administration (FDA) for clinical use, recombinant human bone morphogenetic protein-2 (rhBMP-2) has been widely used in the clinical practice of orthopaedic, including a substantial number of "off label" applications ${ }^{1 ; 2}$. In addition to the approved anterior lumbar interbody fusion, rhBMP-2 is used extensively in other lumbar spinal fusion techniques and at doses that seem to exceed the physiological need ${ }^{3}$. Safety issues pertaining rhBMP-2 have been of concern since the inception of its clinical use. Although many animal and clinical studies suggested that the use of rhBMP-2 in clinical settings should be generally safe ${ }^{4}$, some studies found reasons for concern with respect to uncontrolled bone formation, soft tissue inflammation, and carcinogenesis 5 . In 2011 the FDA denied the approval for a high dose of rhBMP-2 $(40 \mathrm{mg})$ on a compression-resistant hydroxyapatite/ $\beta$-tricalcium phosphate carrier (Amplify, Medtronic, Memphis, TN) for use in posterolateral 
lumbar fusion because of concerns for an increased cancer risk associated with rhBMP- $2^{6}$. A recent publication demonstrated a significant increase of cancer risk in patients treated with high dose rhBMP$2^{7}$, however, two other studies reported different results ${ }^{6 ;} 8$. Controversy continues to the present and no consensus has been reached.

In addition to clinical studies, a large number of basic science studies, both in vitro and in vivo, have been undertaken to investigated the effects of BMP-2 on tumor growth ${ }^{9}$. However, integrating the results of the clinical studies and the basic science studies into a coherent understanding of the relevant processes remains very problematic. Most basic research focused on the direct effects of BMP-2 on different tumor cell types, however, in clinical settings, the systematic carcinogenic effect of BMP-2 is certainly more complex. Animal studies that are more similar to the clinical situation would be helpful to reveal the systematic effect of BMP-2 use. While most in vivo studies for tumor research are performed in mouse models ${ }^{10}$, pre-clinical studies of BMP-2 in orthopaedic applications are mostly done in larger animals, such as rats ${ }^{11}$, rabbits ${ }^{12}$, or canines ${ }^{13}$. A BMP-2 induced spinal fusion model in the mouse would, therefore, provide a much-needed bridge to fill the gap between these two research areas. Although several attempts have been made to establish a mouse spinal fusion model, no standard procedure has been established with detailed protocols and appropriate dosing regimens ${ }^{14 ; 15}$. This study established a posterolateral intertransverse lumbar fusion model in mice using rhBMP-2 by delineating the pertinent anatomy and establishing the optimal dosing regimen for rhBMP-2 in order to validate it as a suitable model to bridge the gap between studies of BMP-2 in bone formation and cancer research.

\section{Materials And Methods.}

This study was performed under a protocol approved by the Animal Research Committee (ARC) of Shanghai Ninth People's Hospital. Recombinant human BMP-2 was obtained from Medtronic (Minneapolis, MN). Forty 10-week-old male Institute of Cancer Research (ICR) mice were maintained and housed in the vivarium in accordance with regulations set forth by the Shanghai Ninth People's Hospital Office of Protection of Research Subjects. Mice were randomly assigned to one of five treatment groups according to the dose of rhBMP-2 that was implanted in association with the surgery, as follows: Group A: control group with carrier collagen sponge only; Group B: $0.05 \mu \mathrm{g}$ rhBMP-2; Group C: $0.15 \mu \mathrm{g}$ rhBMP-2;

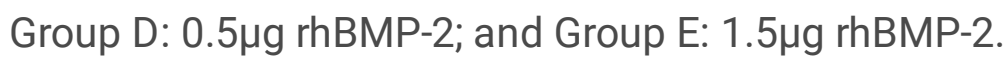

\section{Surgical Procedures}

Posterolateral lumbar fusion surgery was performed in a manner similar to the protocol used for the rat spinal fusion model with which we have considerable experience ${ }^{16}$. Mice were pre-medicated with sustained release buprenorphine thirty minutes prior to surgery. Sodium pentobarbital solution was administered intraperitoneally at the dose of $50 \mathrm{mg} / \mathrm{kg}$. The surgical site, including dorsal aspect of the lumbar spine and iliac crest, was shaved and disinfected with Betadine and $70 \%$ ethanol. The iliac crest was used as a landmark to locate the body of the L6 vertebra. A 2-cm longitudinal midline incision was made through the skin and subcutaneous tissue over L5-L6 down to the lumbodorsal fascia and the 
paraspinal muscles were dissected bilaterally to expose the transverse processes of L5 and L6, which were then decorticated with a high-speed burr. The surgical site was irrigated with sterile saline and $1.5 \mathrm{~mm} \times 1.5 \mathrm{~mm} \times 2 \mathrm{~mm}$ pieces of absorbable collagen sponge (ACS) (Helistat, Integra Life Sciences, Plainsboro, $\mathrm{NJ}$ ) containing the assigned doses of rhBMP-2 were placed bilaterally, with each implant spanning the transverse processes. The ACS, which is made of collagen derived from bovine Achilles tendon, was prepared to deliver the test material by soaking the ACS in a PBS solution of the rhBMP-2 before applying the sponge to the intertransverse process space. The implants were then covered with the overlying paraspinal muscles and the lumbodorsal fascia and skin were closed with 5-0 Prolene sutures (Ethicon, Inc., Somerville, NJ) (Fig 1).

\section{Radiographic Analysis}

Fusion between L5 and L6 was examined by means of radiographs obtained at eight weeks after the surgical procedure. In each mouse, the fusion between the L5 and L6 transverse processes was evaluated by two independent observers, who were blinded with respect to the treatment group assignments, employing the following standardized scale: 0 , no fusion; 1 , unilateral fusion; and 2, complete bilateral fusion. The scores from the observers were added together and only a score of 4 was considered as complete fusion.

\section{Microcomputed Tomography (Micro CT) Analysis}

Mice were euthanized using a standard $\mathrm{CO}_{2}$ chamber eight weeks after the spinal fusion procedure, and their spines were excised and stored in 40\% ethyl alcohol. Each excised spine specimen was analyzed by high resolution microcomputed tomography (micro-CT) using a SkyScan 1172 scanner (SkyScan/Bruker; Kontich, Belgium) as previously described in detai ${ }^{17}$. Virtual image slices were reconstructed using the cone-beam reconstruction software version 2.6 based on the Feldkamp algorithm (SkyScan/Bruker; Kontich, Belgium). Sample reorientation and 2D visualization were performed using Data Viewer (SkyScan/Bruker; Kontich, Belgium), and 3D visualization was performed using Dolphin Imaging version 11 (Dolphin Imaging \& Management Solutions; Chatsworth, CA)

\section{Histology}

The spines were dissected out of the animal, fixed in paraformaldehyde, decalcified in a standard decalcifying solution (Richard-Allan Scientific, Kalamazoo, MI), washed with running tap water, and then transferred to $75 \%$ ethanol ${ }^{18}$. Serial sagittal sections near the transverse processes were cut with a blade carefully at the level of the transverse process. The specimens were embedded in wax and then sectioned. The sections were stained with hematoxylin and eosin, alcian blue, and also alizarin red. The slides were scanned by a Pannoramic SCAN digital slide scanner (3DHISTECH, Budapest, Hungary) using a Zeiss plan-apochromatic objective (magnification: 20x, numerical aperture: 0.8) and a Hitachi (HVF22CL) 3CCD progressive scan color camera (resolution: $0.2325 \mu \mathrm{m} /$ pixel). JPEG image encoding with a quality factor of 80 and an interpolated focus distance of 15 with stitching in the scan options was chosen $^{19}$. 


\section{Statistical Methods}

PASW software (version 18; SPSS, Chicago, Illinois) was used to perform the statistical analyses. The Mann-Whitney $\mathrm{U}$ test was used for comparisons between different groups. Data are expressed as mean \pm SD

\section{Results}

The average weight of the mice was $30.39 \pm 1.89 \mathrm{~g}$, and there were no significant differences among the groups $(P=0.394)$. The mice were allowed to eat and move about ad libitum after surgery. No wound infections or edema were observed in any animal subject. At eight weeks, radiographs of the spines of the mice in the control group showed no new bone formation, while in all of the other treatment groups evidence of new bone formation was observed, although the amount of new bone formation observed in the $0.05 \mu \mathrm{g}$ rhBMP-2 group (Group B) was minimal (Fig. 2). Osseous bridging was detected in Groups C, D and $E$, and complete fusion was seen in Groups $D$ and $E$. Group $E$ showed the most abundant bone formation. The radiographic scores for the degree of fusion increased in relation to the dose of rhBMP-2 and both Groups D and E were given the same score as complete fusion was achieved in both groups (Table 1).

Table 1

Fusion score of different groups

\begin{tabular}{|llllll|}
\hline & Group 1 & Group 2 & Group 3 & Group 4 & Group 5 \\
\hline Observer 1 & 0 & 0.5 & 0.875 & 2 & 2 \\
\hline Observer 2 & 0 & 0.5 & 1 & 2 & 2 \\
\hline
\end{tabular}

In general, the results of the micro-CT analyses were in agreement with those of the plain X-rays. The micro-CT images, however, are more clear and also allow for the amount of new bone formation to be quantified (Fig. 3). The bone volume (BV) in the area of interest (yellow box in Fig. 4A) was measured, and the ratio of bone volume/ total volume (BV/TV) was calculated. Although the $1.5 \mu \mathrm{g}$ of rhBMP-2 group (Group E) resulted in the highest BV compare with other groups, the $0.5 \mu \mathrm{g}$ rhBMP-2 group (Group D) showed better bone quality as reflected in the BV/TV ratio (Fig. 4B,C).

Histology demonstrated that, although the contour of new bone is largest in Group $E$, the inside of the bone shell was occupied mostly by adipocytes. In Group D, on the other hand, the dose of $0.5 \mu \mathrm{g}$ rhBMP-2 induced better bone structure with more trabecular bone inside the tissue bridging the transverse processes (Fig. 5).

\section{Discussion}

BMP-2 belongs to the TGF-beta superfamily of proteins and it can stimulate proliferation in some cell types. Although utilization of BMP-2 can eliminate the associated comorbidities of harvesting allograft, 
use of high-dose rhBMP-2 (> $40 \mathrm{mg}$ ) in orthopaedic surgery should be undertaken cautiously considering its potential adverse effects. A recent publication demonstrated a significant 6.75 -fold increase in the incidence of new cancer two years after single level anterior spinal arthrodesis in patients treated with a high dose of BMP-2 as compared to patients who received autogenous bone grafts ${ }^{20}$. Thus, there is continued concern with respect to carcinogenesis in situations in which exogenous BMP-2 is employed. Nevertheless, in a retrospective study of 502 adult patients who underwent spinal surgery with high-dose BMP-2 the authors reported no correlation between increasing BMP-2 dosage and cancer with an average follow-up period of 42 months ${ }^{21}$. There was, however, a cancer prevalence of $3.4 \%$ in the study patients ${ }^{21}$. Kelly et al. ${ }^{22}$ retrospectively analyzed the incidence of cancer in 467,916 Medicare patients who had undergone spinal arthrodesis between 2005 and 2010. These authors reported no association between the occurrence of cancer and the clinical use of BMP-2.

Basic science studies have also been performed to evaluate the role of rhBMP-2 in the potential stimulation or progression of neoplasms and have reported conflicting findings ${ }^{35-40}$. In tissue culture, BMP-2 stimulates the motility, invasiveness and epithelial-to-mesenchymal transition of osteosarcoma cells $^{23}$. Similar results were reported in pancreatic cancer cells, gastric cancer cells, breast cancer cells and lung cancer cells ${ }^{24-27}$. In mouse xenograft models, some researchers demonstrated that BMP-2 stimulates proliferation and invasiveness of osteosarcoma ${ }^{23 ;} 28$, invasiveness of breast cancer ${ }^{29}$, and angiogenesis in breast and lung cancers ${ }^{30 ;} 31$. On the other hand, Soda et al. ${ }^{32}$ reported BMP-2 did not stimulate proliferation in human breast cancer cells, ovarian cancer cells or prostate cancer cells. And furthermore, Ye et al. ${ }^{33}$ observed that BMP-2 had an inhibitory effect on breast cancer cell growth in a nude mice model and Zhao et al. ${ }^{34}$ reported that BMP-2 inhibited mouse GNP-like medulloblastoma growth in vivo.

In addition to the conflicting results of these basic science studies, the consideration remains that most of these studies focused on the direct effect of BMP-2 on tumor growth, whereas, in clinical settings, BMP-2 is usually administrated in a normal area and the cancer risk is associated with an effect at a distant site. Animal studies that more closely resemble the actual clinical situation would be helpful to address this short-coming. Most in vivo studies for tumor research are conducted in mouse models and, therefore, a mouse spinal fusion model involving BMP-2 will eliminate a substantial barrier to progress in this research. To date, larger animals such as rats, rabbits, and canines have been used for spinal fusion models as it was thought that mice are too small for such surgical procedures. We have now demonstrated that this surgical procedure can effectively and reliably be accomplished. In this study we determined the optimal dose of rhBMP-2. A dose of $0.5 \mu \mathrm{g}$ provided for the highest fusion rate with the highest bone quality and, therefore, it is our recommendation that this dose be employed in future studies.

\section{Conclusion}


This is the first study to introduce a standardized procedure for a BMP-2 induced posterolateral intertransverse lumbar fusion in mice. The procedure is not excessively challenging from the technical point of view and the results are reliable. The optimal dose for rhBMP-2 is $0.5 \mu \mathrm{g}$. Future studies can now include this procedure in studies on the potential carcinogenicity of rhBMP-2.

\section{Abbreviations}

rhBMP-2: recombinant human bone morphogenetic protein-2

ICR: Institute of Cancer Research

FDA: Food and Drug Administration

ARC: Animal Research Committee

ACS: absorbable collagen sponge

Micro CT: Microcomputed Tomography

\section{Declarations}

Ethics approval and consent to participate

This study has been approved by the institutional review board of Shanghai Ninth People's Hospital.

\section{Consent for publication}

Not applicable.

\section{Availability of data and materials}

The datasets used and/or analysed during the current study are available from the corresponding author on reasonable request.

\section{Competing interests}

Disclosures for Jeffrey C. Wang, MD

Royalties - Biomet, Seaspine, Amedica, Synthes

Investments/Options - Bone Biologics, Pearldiver, Electrocore, Surgitech

Board of Directors - North American Spine Society, AO Foundation, Cervical Spine Research Society, Society for Brain Mapping and Therapeutics, American Orthopaedic Association

Editorial Boards - Spine, The Spine Journal, Clinical Spine Surgery, Global Spine Journal 
Fellowship Funding (paid to institution): AO Foundation

Dr Michael D. Daubs receive royalties and is a paid consultant for the orthopaedic product or device: Dupuy-Synthes. Dr Michael D. Daubs receive research or institutional support as a principal investigator from the orthopaedic device company Stryker

\section{Funding}

This work was supported by National Natural Science Foundation of China (Grant No. 81402229), China Postdoctoral Science Foundation (Grant No. 2017M611584, 2018T110400). We declare that we do not have any commercial or associative interest that represents a conflict of interest in connection with the work submitted.

\section{Authors' contributions}

Design: HT, CZ, JW, MD, SM and EB. Experimental operation: HC, LD, CH and HT. Acquisition of data and Analysis: $H C, H T, M G$. Writing, review, and/or revision of the manuscript: $H T, H C, M G, L D, C H, C Z, E B, M D$, JW and SM. All authors read and approved the final manuscript.

\section{Acknowledgements}

Not applicable.

\section{References}

1. Cheung A, Phillips AM. 2006. Bone morphogenetic proteins in orthopaedic surgery. Curr Orthop 20:424-429.

2. Axelrad TW, Einhorn TA. 2009. Bone morphogenetic proteins in orthopaedic surgery. Cytokine \& growth factor reviews 20:481-488.

3. Esmail N, Buser Z, Cohen JR, et al. 2018. Postoperative Complications Associated With rhBMP2 Use in Posterior/Posterolateral Lumbar Fusion. Global spine journal 8:142-148.

4. Poynton AR, Lane JM. 2002. Safety profile for the clinical use of bone morphogenetic proteins in the spine. Spine 27:S40-48.

5. Tian H, Zhao J, Brochmann EJ, et al. 2017. Bone morphogenetic protein-2 and tumor growth: Diverse effects and possibilities for therapy. Cytokine \& growth factor reviews 34:73-91.

6. Mesfin A, Buchowski JM, Zebala LP, et al. 2013. High-dose rhBMP-2 for adults: major and minor complications: a study of 502 spine cases. The Journal of bone and joint surgery American volume 95:1546-1553.

7. Carragee EJ, Chu G, Rohatgi R, et al. 2013. Cancer risk after use of recombinant bone morphogenetic protein-2 for spinal arthrodesis. The Journal of bone and joint surgery American volume 95:15371545. 
8. Kelly MP, Savage JW, Bentzen SM, et al. 2014. Cancer risk from bone morphogenetic protein exposure in spinal arthrodesis. The Journal of bone and joint surgery American volume 96:14171422.

9. Skovrlj B, Koehler SM, Anderson PA, et al. 2015. Association Between BMP-2 and Carcinogenicity. Spine 40:1862-1871.

10. Bock BC, Stein U, Schmitt CA, et al. 2014. Mouse models of human cancer. Cancer research 74:46714675.

11. Kaito T, Johnson J, Ellerman J, et al. 2013. Synergistic effect of bone morphogenetic proteins 2 and 7 by ex vivo gene therapy in a rat spinal fusion model. The Journal of bone and joint surgery American volume 95:1612-1619.

12. Scott TP, Phan KH, Tian H, et al. 2015. Comparison of a novel oxysterol molecule and rhBMP2 fusion rates in a rabbit posterolateral lumbar spine model. The spine journal : official journal of the North American Spine Society 15:733-742.

13. David SM, Gruber HE, Meyer RA, Jr., et al. 1999. Lumbar spinal fusion using recombinant human bone morphogenetic protein in the canine. A comparison of three dosages and two carriers. Spine 24:1973-1979.

14. Rao RD, Bagaria VB, Cooley BC. 2007. Posterolateral intertransverse lumbar fusion in a mouse model: surgical anatomy and operative technique. The spine journal : official journal of the North American Spine Society 7:61-67.

15. Bobyn J, Rasch A, Little DG, et al. 2013. Posterolateral inter-transverse lumbar fusion in a mouse model. Journal of orthopaedic surgery and research 8:2.

16. Montgomery SR, Nargizyan T, Meliton V, et al. 2014. A novel osteogenic oxysterol compound for therapeutic development to promote bone growth: activation of hedgehog signaling and osteogenesis through smoothened binding. Journal of bone and mineral research : the official journal of the American Society for Bone and Mineral Research 29:1872-1885.

17. Tian H, Li CS, Zhao KW, et al. 2015. A carboxy terminal BMP/TGF-beta binding site in secreted phosphoprotein $24 \mathrm{kD}$ independently affects BMP-2 activity. Journal of cellular biochemistry 116:667-676.

18. Tian H, Li CS, Scott TP, et al. 2015. Secreted phosphoprotein 24 kD inhibits nerve root inflammation induced by bone morphogenetic protein-2. The spine journal : official journal of the North American Spine Society 15:314-321.

19. Marien KM, Croons V, Waumans Y, et al. 2016. Development and Validation of a Histological Method to Measure Microvessel Density in Whole-Slide Images of Cancer Tissue. PloS one 11:e0161496.

20. Carragee EJ, Gilbert C, Rajat R, et al. 2013. Cancer risk after use of recombinant bone morphogenetic protein-2 for spinal arthrodesis. Journal of Bone \& Joint Surgery-american Volume 95:1537-1545.

21. Addisu M, Buchowski JM, Zebala LP, et al. 2013. High-dose rhBMP-2 for adults: major and minor complications: a study of 502 spine cases. Journal of Bone \& Joint Surgery-american Volume 95:1546-1553. 
22. Kelly MP, Savage JW, Bentzen SRM, et al. 2014. Cancer risk from bone morphogenetic protein exposure in spinal arthrodesis. Journal of Bone \& Joint Surgery-american Volume 96:1417-1422.

23. Tian H, Zhou T, Chen H, et al. 2019. Bone morphogenetic protein-2 promotes osteosarcoma growth by promoting epithelial-mesenchymal transition (EMT) through the Wnt/beta-catenin signaling pathway. Journal of orthopaedic research : official publication of the Orthopaedic Research Society.

24. Xiong C, Jie L, Yebin L, et al. 2011. Activation of the PI3K/Akt pathway mediates bone morphogenetic protein 2-induced invasion of pancreatic cancer cells Panc-1. Pathology \& Oncology Research 17:257-261.

25. Gordon KJ, Kirkbride KC, Tam H, et al. 2009. Bone morphogenetic proteins induce pancreatic cancer cell invasiveness through a Smad1-dependent mechanism that involves matrix metalloproteinase-2. Carcinogenesis 30:238-248.

26. Ya-Ling H, Ming-Shyan H, Chih-Jen Y, et al. 2011. Lung tumor-associated osteoblast-derived bone morphogenetic protein-2 increased epithelial-to-mesenchymal transition of cancer by Runx2/Snail signaling pathway. Journal of Biological Chemistry 286:37335-37346.

27. Kang MH, Kim JS, Ji ES, et al. 2010. BMP2 accelerates the motility and invasiveness of gastric cancer cells via activation of the phosphatidylinositol 3-kinase (PI3K)/Akt pathway. Experimental Cell Research 316:24-37.

28. Guo W, Gorlick R, M, Meyers P, et al. 1999. Expression of bone morphogenetic proteins and receptors in sarcomas. Clinical Orthopaedics \& Related Research 365:175-183.

29. Clement JH, Raida M, Sänger J, et al. 2005. Bone morphogenetic protein 2 (BMP-2) induces in vitro invasion and in vivo hormone independent growth of breast carcinoma cells. International Journal of Oncology 27:401.

30. Langenfeld EM, Langenfeld J. 2004. Bone morphogenetic protein-2 stimulates angiogenesis in developing tumors. Molecular Cancer Research Mcr 2:141.

31. Raida M, Clement JH, Leek RD, et al. 2005. Bone morphogenetic protein 2 (BMP-2) and induction of tumor angiogenesis. Journal of Cancer Research \& Clinical Oncology 131:741-750.

32. Soda H, ., Raymond E, ., Sharma S, ., et al. 1998. Antiproliferative effects of recombinant human bone morphogenetic protein-2 on human tumor colony-forming units. Anti-Cancer Drugs 9:327-331.

33. Shuai Y, Byung-Hyun P, Kyung-Jin S, et al. 2013. In vivo inhibition of bone morphogenetic protein-2 on breast cancer cell growth. Spine 38:E143-E150.

34. Haotian Z, Olivier A, Frederique Z, et al. 2008. Post-transcriptional down-regulation of Atoh1/Math1 by bone morphogenic proteins suppresses medulloblastoma development. Genes \& Development 22:722-727.

\section{Figures}




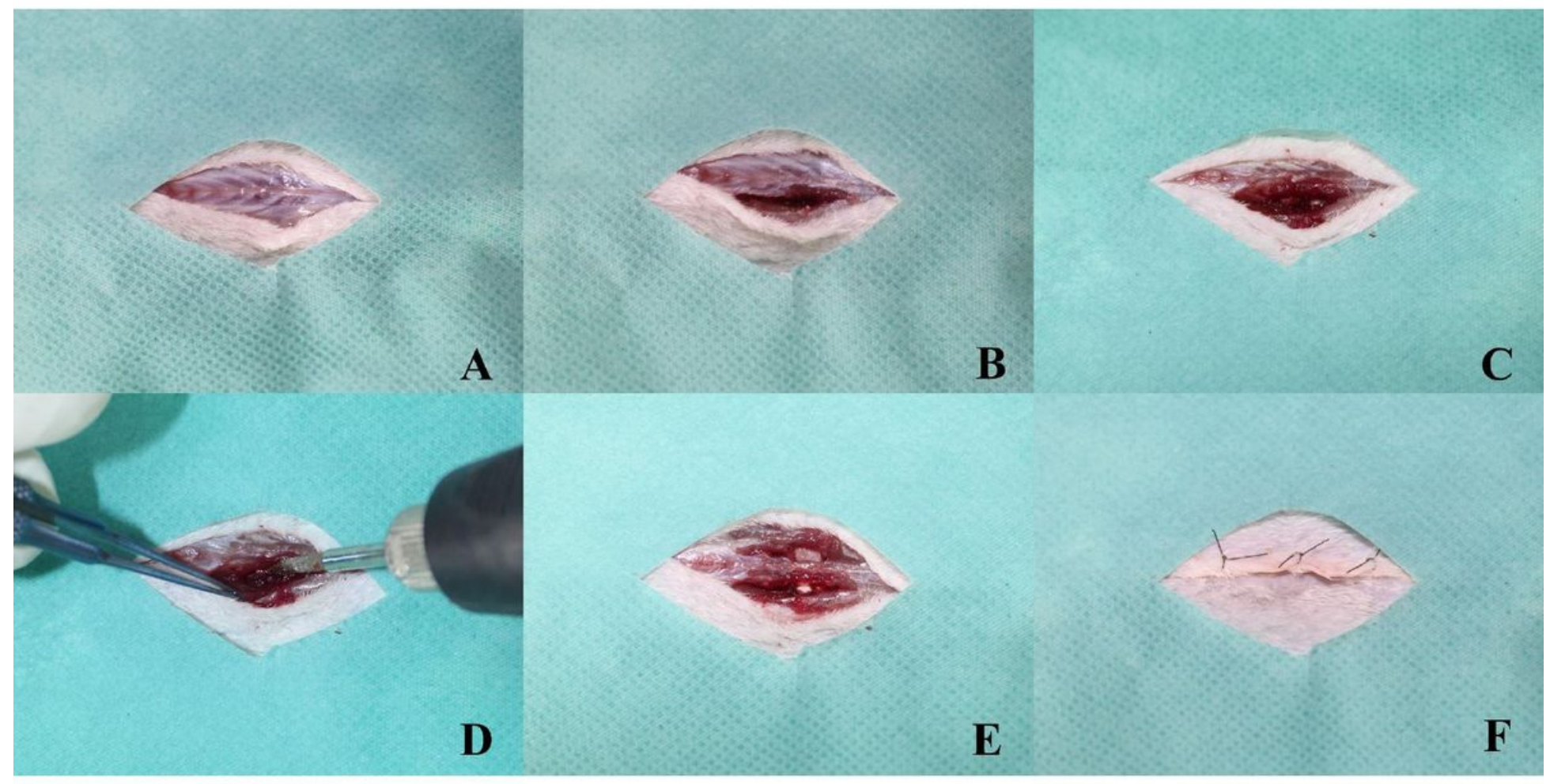

\section{Figure 1}

Surgical procedure of the posterolateral intertransverse lumbar fusion model using mice. A, a 2-cm longitudinal midline skin incision was made over L5-L6 ; B, soft tissue was dissected down to the lumbodorsal fascia; $\mathrm{C}$, the paraspinal muscles were dissected bilaterally to expose the transverse processes of L5-L6; $D$, the transverse processes were decorticated with a high-speed burr; $E$, absorbable collagen sponge (ACS) (Helistat, Integra Life Sciences) containing different doses of rhBMP-2 were placed bilaterally to the intertransverse process space; $F$, the incision was closed with 5-0 Prolene sutures (Ethicon, Inc., Somerville, NJ).

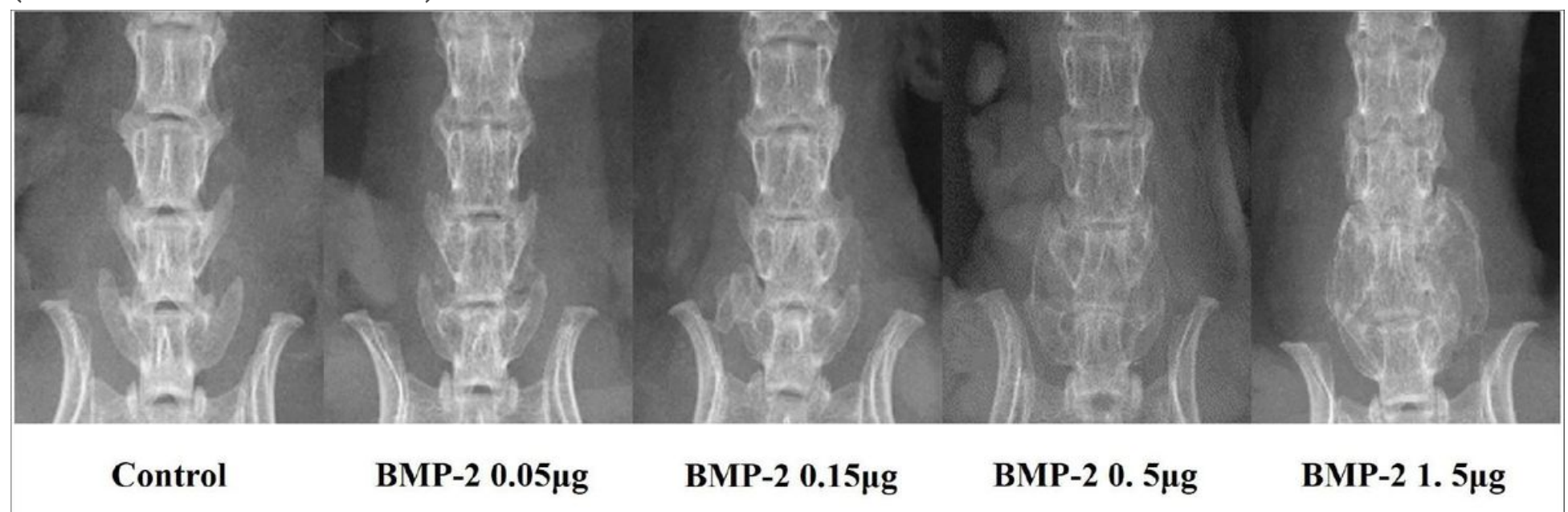

Figure 2 
Radiographs of the spines of the mice at eight weeks. Control group showed no new bone formation; minimal bone formation in the $0.05 \mu \mathrm{g}$ rhBMP-2 group; osseous bridging was detected in Groups C; Groups D, E appeared complete fusion.

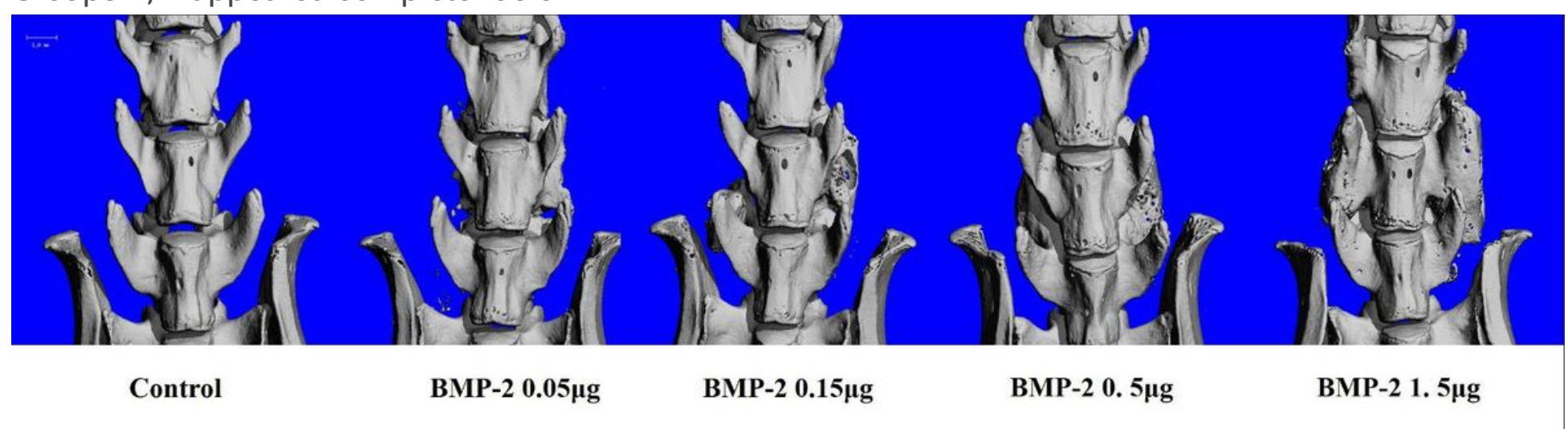

\section{Figure 3}

Images of the fusion segments reconstructed from micro-CT data.

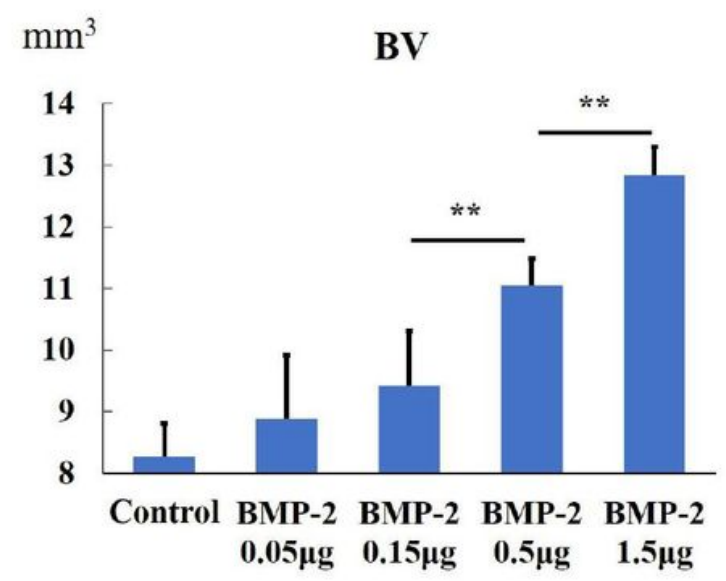

A

B

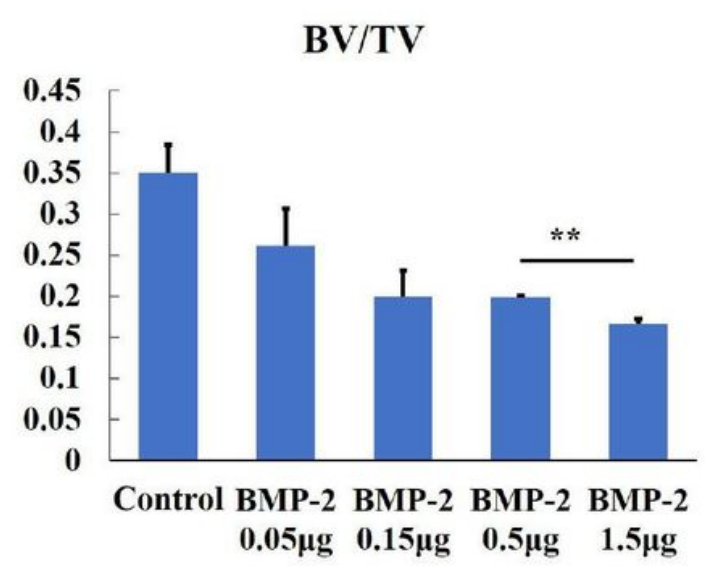

C

$* *: P<0.01$

\section{Figure 4}

Micro-CT data analysis of the fusion segments. A, The bone volume (BV) was measured in the area of interest (yellow box); B, BV was compared between groups; $\mathrm{C}$, the ratio of bone volume/ total volume (BV/TV) was calculated and compared among groups. 


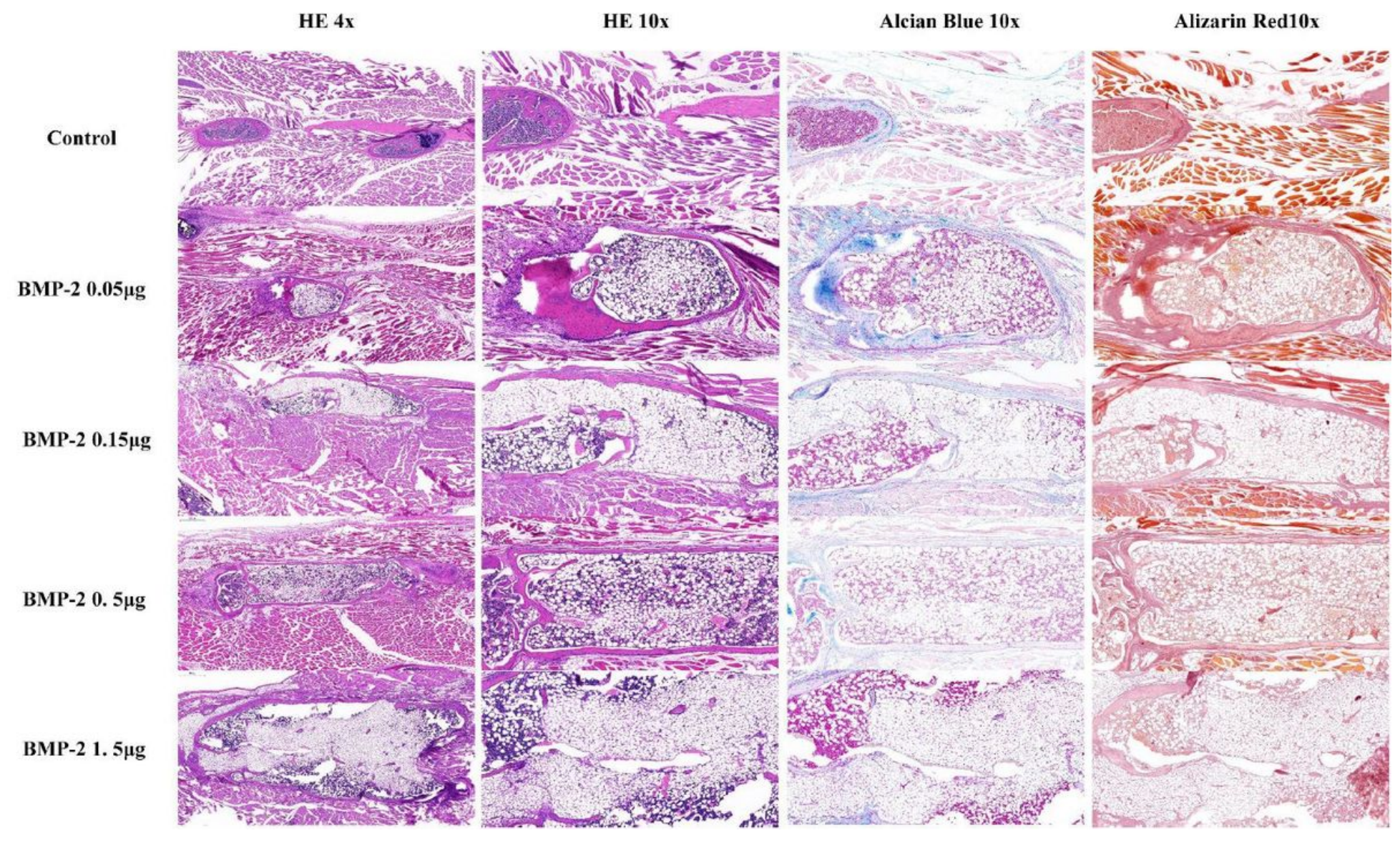

Figure 5

Histology of the fusion segments. Though the largest contour of new bone was observed in the $1.5 \mu \mathrm{g}$ rhBMP-2 group, inside the bone shell was mostly adipose cells. The $0.5 \mu \mathrm{g}$ rhBMP-2 group showed better bone structure with more trabecular bone inside. 